\title{
Are stop codons recognized by base triplets in the large ribosomal RNA subunit?
}

\author{
HAN LIANG, ${ }^{1}$ LAURA F. LANDWEBER, ${ }^{2}$ and JACQUES R. FRESCO ${ }^{3}$ \\ Departments of Chemistry, ${ }^{1}$ Ecology \& Evolutionary Biology, ${ }^{2}$ and Molecular Biology, ${ }^{3}$ Princeton University, Princeton, \\ New Jersey 08544, USA
}

\begin{abstract}
The precise mechanism of stop codon recognition in translation termination is still unclear. A previously published study by Ivanov and colleagues proposed a new model for stop codon recognition in which 3-nucleotide Ter-anticodons within the loops of hairpin helices 69 (domain IV) and 89 (domain V) in large ribosomal subunit (LSU) rRNA recognize stop codons to terminate protein translation in eubacteria and certain organelles. We evaluated this model by extensive bioinformatic analysis of stop codons and their putative corresponding Ter-anticodons across a much wider range of species, and found many cases for which it cannot explain the stop codon usage without requiring the involvement of one or more of the eight possible noncomplementary base pairs. Involvement of such base pairs may not be structurally or thermodynamically damaging to the model. However, if, according to the model, Ter-anticodon interaction with stop codons occurs within the ribosomal A-site, the structural stringency which that site imposes on sense codon·tRNA anticodon interaction should also extend to stop codon-Ter-anticodon interactions. Moreover, with Ter-tRNA in place of an aminoacyl-tRNA, for each of the various Ter-anticodons there is a sense codon that can interact with it preferentially by complementary and wobble base-pairing. Both these considerations considerably weaken the arguments put forth previously.
\end{abstract}

Keywords: translation termination; stop codon usage; stop codon recognition; putative rRNA anticodons; mitochondrial genetic code

\section{INTRODUCTION}

Protein translation is a key cellular process. With protein sequences encoded in messenger RNA sequences, termination of the synthesis of any particular protein begins with the recognition of a stop codon in the message by some component(s) of the translation machinery. While the basic scheme for translation of the message has been known for many years, the precise way in which stop codon recognition occurs remains elusive (Nakamura and Ito 1998).

Recently, Ivanov et al. (2001) presented a new model for recognition of stop codons. They noted that hairpin helices 69 (domain IV) and 89 (domain V) in the rRNA of the large ribosomal subunit (LSU) of Escherichia coli, with 7-nucleotide (nt) hairpin loops (the same size as the anticodon loop of tRNA), contain CUA or UCA triplets in the middle of the loop, just like the anticodons in tRNA. These two triplets are antiparallel complements of the stop codons in E. coli, as if

Reprint requests to: Jacques R. Fresco, Department of Molecular Biology, Princeton University, Princeton, NJ 08544, USA; e-mail: jfresco@ molbio.princeton.edu; or Laura Landweber, Department of Ecology \& Evolutionary Biology, Princeton University, Princeton, NJ 08544, USA; e-mail: 1fl@princeton.edu.

Article and publication are at http://www.rnajournal.org/cgi/doi/10.1261/ rna.2780505 they were their corresponding tRNA anticodons. For this reason, and because of the similarity between the anticodon stem and loop in tRNA and these two proposed ribosomal mimics, Ivanov et al. termed hairpins 69 and 89 termination tRNA1 (Ter-tRNA1) and termination tRNA2 (Ter-tRNA2), respectively. This terminology was introduced without any implication that tRNAs with stop-anticodons actually exist. Ivanov and colleagues conjectured that eubacteria and certain organelles (mitochondria and chloroplasts) recognize stop codons in mRNA by the so-called Ter-tRNA anticodons of the tRNA mimics within the LSU rRNA. Specifically, they proposed that Ter-tRNA1, containing "anticodon" CUA, recognizes both UAG and UAA stop codons, whereas TertRNA2, containing "anticodon" UCA, recognizes UGA and UAA. This left the role of the protein release factors RF1 and RF2 to that of promoting the accuracy of stop codon-Teranticodon recognition. As supportive evidence for the generality of the model, Ivanov and colleagues noted that vertebrate mitochondria, which utilize two different stop codons, AGA and AGG, contain the corresponding stop Ter-anticodons, UCU and CCU, in the loops of hairpins 69 and 89 of the LSU rRNA. Armed with this example of a possible compensatory change, they implied that these mitochondria most likely conserve the Ter-tRNA mechanism to recognize their stop codons. 
The idea of stop codon recognition by a complementary triplet in the LSU rRNA does not conflict with the wellknown "tripeptide anticodon" model, in which the tripeptide motifs of RF1 and RF2 are viewed as functionally equivalent to the tRNA anticodons and respond specifically to stop codons (Ito et al. 2000). As Ivanov et al. (2001) proposed, it is possible that RF1 and RF2 recognize the miniduplex formed by stop codon interaction with the anticodon of Ter-tRNA rather than the stop codon alone. Therefore, additional studies are required to determine whether the proposed Ter-tRNA anticodons are actually involved in stop codon recognition.

To test the Ivanov model, we examined a wide range of species for the relation between their stop codon usage and their potential Ter-anticodon sequences. Noticing particularly that many mitochondria and some eubacteria (e.g., Firmicutes) do not employ standard stop codons, we even considered possible alternative base pairs that might be compatible with the basic model of Ivanov et al. without requiring Watson-Crick stop codon-LSU Ter-anticodon complementarity.

\section{RESULTS}

We first summarize our more extensive analysis of stop codons and potential Ter-anticodons, and then evaluate whether the base-pairing possibilities required by the model of Ivanov et al. are structurally and thermodynamically plausible and consistent with the specificity requirements of the model. We then consider several additional observations that our data mining uncovered, and conclude with an overall evaluation of the Ter-tRNA model.

\section{Analysis of relevant sequences in mitochondrial rRNAs}

The extensive variation in stop codon usage in mitochondria provides considerable opportunity to test the Ivanov model. We divided the mitochondrial LSU rRNAs (comparative RNA web, Cannone et al. 2002) into four groups according to stop codon usage. We considered only stop codon usage confirmed by gene annotation, and included cases with both standard and unusual stop codons.

Of the 242 mitochondrial species examined, 12 use the standard stop codons UAG, UAA, and UGA; and most of these have Ter-anticodons in hairpins 69 and 89 with noncomplementary residues. For example, although the model predicts that hairpin 69 Ter-anticodons should recognize UAG/UAA and hairpin 89 Ter-anticodons should recognize UAA/UGA, 10 species have a putative Ter-anticodon in hairpin 69 of $5^{\prime}$-CUC- $3^{\prime}$ or $5^{\prime}$-CUA-3', whereas those in hairpin 89 include $5^{\prime}$-ACC-3', 5'-UCA- $3^{\prime}, 5^{\prime}$-UCC- $3^{\prime}, 5^{\prime}$ UCU- $3^{\prime}$, and $5^{\prime}$-CCC- $3^{\prime}$ (Table $1 \mathrm{~A}$ ). We therefore consider in the next section whether the implied noncomplementary base-pairing interactions are structurally and thermodynamically plausible.

We also found 73 species comprising a second group that uses only UAA and UAG as stop codons, since UGA is reassigned to tryptophan. Only hairpin 69 matters in this group, as it recognizes UAA and UAG according to

TABLE 1. Examples of noncomplementary base pairs required by the Ter-tRNA model in mitochondrial LSU rRNA loop sequences of hairpins 69 and 89 for (A) the standard stop codon group, (B) the UAR stop codon group, (C) the Vertebrate Mitochondria Stop Codon Group, and (D) two protists, Thraustochytrium aureum and Scenedesmus obliquus, with unique mitochondrial stop codon usage. Only hairpin 69 is considered in cases where UGA is a sense codon (B and C). Ter-anticodons are uppercase letters of loop sequences

\begin{tabular}{|c|c|c|c|c|c|c|}
\hline \multicolumn{7}{|l|}{ A } \\
\hline \multirow[b]{4}{*}{ Species } & \multicolumn{3}{|c|}{ Hairpin 69} & \multicolumn{3}{|c|}{ Hairpin 89} \\
\hline & \multirow[b]{3}{*}{$\begin{array}{l}\text { Ter-tRNA1 loop } \\
\text { sequence }\end{array}$} & \multicolumn{2}{|c|}{ Stop codon } & \multirow[b]{3}{*}{$\begin{array}{l}\text { Ter-tRNA2 loop } \\
\text { sequence }\end{array}$} & \multicolumn{2}{|c|}{ Stop codon } \\
\hline & & UAG & UAA & & UGA & UAA \\
\hline & & \multicolumn{2}{|c|}{$\begin{array}{c}\text { Noncomplementary } \\
\text { base pairs }\end{array}$} & & \multicolumn{2}{|c|}{$\begin{array}{c}\text { Noncomplementary } \\
\text { base pairs }\end{array}$} \\
\hline Physarum polycephalum & $5^{\prime}$-aaCUAug-3' & - & A.C & $5^{\prime}$-guACCua-3' & $C \cdot U A \cdot A$ & $C \cdot U$ A.C A.A \\
\hline Chlamydomonas eugametos & $5^{\prime}$-aaCUCua-3' & $\mathrm{C} \cdot \mathrm{U}$ & $C \cdot U A \cdot C$ & $5^{\prime}$-guUCAua-3' & X & A.C \\
\hline Chlamydomonas reinhardtii & $5^{\prime}$-aaCUCua-3' & $C \cdot U$ & $C \cdot \cup A \cdot C$ & 5'-guUCCua-3' & $x$ & $C \cdot U A \cdot C$ \\
\hline Nephroselmis olivacea & $5^{\prime}$-aaCUCua-3' & $C \cdot U$ & $C \cdot U A \cdot C$ & $5^{\prime}$-gcUCUua-3' & $x$ & $U \cdot U A \cdot C$ \\
\hline Marchantia polymorpha & $5^{\prime}$-aaCUCua-3' & $C \cdot U$ & $C \cdot U A \cdot C$ & 5'-gcUCUua-3' & $U \cdot U$ & $U \cdot U A \cdot C$ \\
\hline Triticum aestivum & $5^{\prime}$-aaCUCua-3' & $C \cdot U$ & $C \cdot \cup A \cdot C$ & $5^{\prime}$-gcUCUua-3' & $U \cdot U$ & $U \cdot U A \cdot C$ \\
\hline Arabidopsis thaliana & $5^{\prime}$-aaCUCua-3' & $\mathrm{C} \cdot \mathrm{U}$ & $C \cdot U A \cdot C$ & $5^{\prime}$-gcUCUua-3' & $U \cdot U$ & $U \cdot U A \cdot C$ \\
\hline Zea mays & $5^{\prime}$-aaCUCua- $3^{\prime}$ & $\mathrm{C} \cdot \mathrm{U}$ & $C \cdot U A \cdot C$ & $5^{\prime}$-gc UCUua-3' & $U \cdot U$ & U.U A.C \\
\hline Oenothera berteriana & $5^{\prime}$-aaCUCua- $3^{\prime}$ & $\mathrm{C} \cdot \mathrm{U}$ & $C \cdot U A \cdot C$ & $5^{\prime}$-gcUCUua-3' & $U \cdot U$ & $U \cdot U A \cdot C$ \\
\hline Pilayella littoralis & $5^{\prime}$-aaCUAug-3' & - & A.C & $5^{\prime}$-guCCCua-3' & $C \cdot U A \cdot C$ & $C \cdot U A \cdot C$ \\
\hline
\end{tabular}


TABLE 1. Continued

B

\begin{tabular}{|c|c|c|c|c|}
\hline \multirow[b]{4}{*}{ Species } & \multicolumn{4}{|c|}{ Hairpin 69} \\
\hline & \multirow[b]{3}{*}{$\begin{array}{l}\text { Ter-tRNA1 loop } \\
\text { sequence }^{\mathrm{a}}\end{array}$} & \multirow[b]{3}{*}{$\begin{array}{c}\text { Alternate } \\
\text { ter-anticodons }{ }^{\mathrm{b}}\end{array}$} & \multicolumn{2}{|c|}{ Stop codon } \\
\hline & & & UAA & UAG \\
\hline & & & \multicolumn{2}{|c|}{$\begin{array}{c}\text { Noncomplementary } \\
\text { base pairs }\end{array}$} \\
\hline Neurospora crassa & $5^{\prime}$-gUAAAg- $3^{\prime}$ & UAA/AAA & $A \cdot A / A \cdot A$ & G.U A.A/G.A A.A \\
\hline Podospora anserina & $5^{\prime}$-aGCGUg-3' & GCG/CGU & A.G A.C G.U/A.C A.G U.U & $G \cdot G$ A.C U.G/A.G U.U \\
\hline Pecten maximus & $5^{\prime}-\mathrm{aGCGUg-3^{ \prime }}$ & GCG/CGU & " & $"$ \\
\hline Ascaris suum & $5^{\prime}$-aGCGUg- $3^{\prime}$ & GCG/CGU & " & " \\
\hline Caenorhabditis elegans & $5^{\prime}$-aGCGUg-3' & GCG/CGU & " & " \\
\hline Onchocerca volvulus & $5^{\prime}$-aGCGUg-3' & GCG/CGU & " & " \\
\hline Meloidogyne hapla & $5^{\prime}$-aGCGUg-3' & GCG/CGU & " & " \\
\hline Meloidogyne javanica & $5^{\prime}$-aGCGUg-3' & GCG/CGU & " & " \\
\hline
\end{tabular}

C

\begin{tabular}{|c|c|c|c|c|c|}
\hline \multirow[b]{5}{*}{ Species } & \multicolumn{5}{|c|}{ Hairpin 69} \\
\hline & \multirow[b]{4}{*}{$\begin{array}{l}\text { Ter-tRNA1 loop } \\
\text { sequence }^{c}\end{array}$} & \multicolumn{4}{|c|}{ Stop codon } \\
\hline & & \multicolumn{2}{|c|}{ New } & \multicolumn{2}{|c|}{ Old } \\
\hline & & AGA & AGG & UAA & UAG \\
\hline & & \multicolumn{4}{|c|}{$\begin{array}{c}\text { Noncomplementary } \\
\text { base pairs }\end{array}$} \\
\hline Raja radiata & $5^{\prime}$-auUAGg- $3^{\prime}$ & A.G & $A \cdot G \mathrm{G} \cdot U$ & $G \cdot \cup A \cdot G A \cdot A$ & G.U A.G \\
\hline Rattus norvegicus & $5^{\prime}$-guAAAg-3' & $A \cdot A \cdot A \cdot G$ & $A \cdot A \cdot A \cdot G$ & A.A G.U & $G \cdot \cup A \cdot A$ A.G \\
\hline
\end{tabular}

D

\begin{tabular}{|c|c|c|c|c|c|c|c|c|c|c|}
\hline \multirow[b]{4}{*}{ Species } & \multicolumn{5}{|c|}{ Hairpin 69} & \multicolumn{5}{|c|}{ Hairpin 89} \\
\hline & \multirow[b]{3}{*}{$\begin{array}{c}\text { Ter-tRNA1 } \\
\text { loop sequence }\end{array}$} & \multicolumn{4}{|c|}{ Stop codon } & \multirow[b]{3}{*}{$\begin{array}{c}\text { Ter-tRNA2 } \\
\text { loop sequence }\end{array}$} & \multicolumn{4}{|c|}{ Stop codon } \\
\hline & & UAG & UAA & UUA & UCA & & UAA & UGA & UUA & UCA \\
\hline & & \multicolumn{4}{|c|}{$\begin{array}{c}\text { Noncomplementary } \\
\text { base pairs }\end{array}$} & & \multicolumn{4}{|c|}{$\begin{array}{c}\text { Noncomplementary } \\
\text { base pairs }\end{array}$} \\
\hline T. aureum & $5^{\prime}$-aaCUAua-3' & - & A.C & $A \cdot C \cup \cdot U$ & X & $5^{\prime}$-guUCAua-3' & A.C & - & $C \cdot U$ & X \\
\hline S. obliquus & 5'-aaCUCua-3' & $X$ & $\mathrm{~A} \cdot \mathrm{C} \mathrm{C} \cdot \mathrm{U}$ & X & $\mathrm{A} \cdot \mathrm{C} \mathrm{C} \cdot \mathrm{U}$ & $5^{\prime}$-guUCCaa-3' & $\mathrm{A} \cdot \mathrm{C} \mathrm{C} \cdot \mathrm{U}$ & $\mathrm{C} \cdot \mathrm{U}$ & $x$ & $\mathrm{C} \cdot \mathrm{C} \mathrm{C} \cdot \mathrm{U}$ \\
\hline
\end{tabular}

${ }^{a}$ Overlapping Ter-anticodons are shown in uppercase letters of loop sequences.

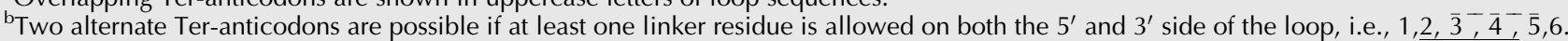

'New Ter-anticodons (nucleotides 3, 4, 5 in uppercase letters, corresponding to new stop codons AGR) overlap old ones (nucleotides 4, 5, 6, corresponding to old stop codons UAR).

(-) No noncomplementary base pairs.

(X) Stop codon absent from this species.

the model. Among these Ter-anticodons are base triplets that again imply noncomplementary base-pairing interactions. Also, in some species, hairpin 69 has a 6-nt instead of a 7-nt loop normally present in the anticodon loop of tRNA. This makes the location of the Ter-anticodon ambiguous. In tRNA, the anticodon loop contains $2 \mathrm{nt}$ on either side of the anticodon triplet that presumably give the anticodon unrestricted interaction with the codon triplet of the message. In a similar but restricted manner, we permit at least $1 \mathrm{nt}$ in hairpin 69 to serve as a linker on both sides of the Ter-anticodon. Therefore, we consider two alternative triplets, either positions $2-4$ or $3-5$ of the 6 -nt loop, counting the $5^{\prime}$ position of the loop as nt 1 . By this flexible criterion, there are still eight cases in which the stop codon-Ter-anticodon duplex cannot form by complementary base-pairing (Table 1B). 
Another 155 species comprise a Vertebrate Mitochondria Stop Codon Group using UAG, UAA, AGA, and AGG as stop codons. In Ivanov et al. (2001), vertebrate mitochondria were taken to indicate strong support for their model because the "new" AGG and AGA stop codons match CCU or UCU Ter-anticodons in hairpin 69, whereas triplets CUA or CUG at the $3^{\prime}$ end of the hairpin loop are complementary to the "old" stop codons UAG and UAA. This implies that one linker residue was deleted from the $3^{\prime}$ end of the loop, reducing the loop size to 6 nt. However, our search uncovered two exceptions (Table 1C) in Raja radiata and Rattus norvegicus that imply noncomplementary base pairs. Ivanov and colleagues also explained the presence of the Ter-anticodon UUU, positioned at nt 3-5 in hairpin 69 of fish mitochondrial LSU rRNA, by noting that the AGG and AGA stop codons are absent from these species. However, we found several closely related species (Table 2) with the same sequence in the loop of hairpin 69, that nevertheless do use AGA or AGG as stop codons. This would require G.U base pairs in the second or both the second and third codon positions.

Two protists, the stramenopile Thraustochytrium aureum and green algae Scenedesmus obliquus, with "new" stop codons UUA and UCA, respectively, also require noncomplementary base-pairing with the corresponding Ter-anticodons at either hairpin site (Table 1D). Although a majority of genes in both species use new stop codons, the Ter-anticodons cannot pair conventionally with the two "old" stop codons in these cases.

In sum, we found that the Ter-tRNA model requires noncomplementary base pairs for each stop codon group. Therefore, we further tested the model by evaluating the feasibility of noncomplementary base pairs within the context of the ribosome.

\section{Analysis of noncomplementary base pairs}

The four canonical bases conceivably give rise to 10 unique base pairs (Fig. 1). The cases cited above require every

TABLE 2. Exceptions in the Vertebrate Mitochondrial Stop Codon Group that use AGA and AGG in addition to the UAA and UAG stop codons

\begin{tabular}{lcc}
\hline & \multicolumn{2}{c}{ Number of atypical stop codons in genome } \\
\cline { 2 - 3 } Species & AGA & AGG \\
\hline Mustelus manazo & 0 & 1 \\
Gadus morhua & 2 & 0 \\
Neoceratodus foresteri & 1 & 0 \\
Lepidosiren paradoxa & 1 & 0 \\
Protopterus dolloi & 0 & 1 \\
Petromyzon marinus & 6 & 0 \\
\hline
\end{tabular}

The species shown all contain the sequence $5^{\prime}$-auUUUg- $3^{\prime}$ in hairpin 69, with UUU representing the Ter-anticodon for new stop codons UAR, requiring G.U wobble pairs at second and third stop codon positions.
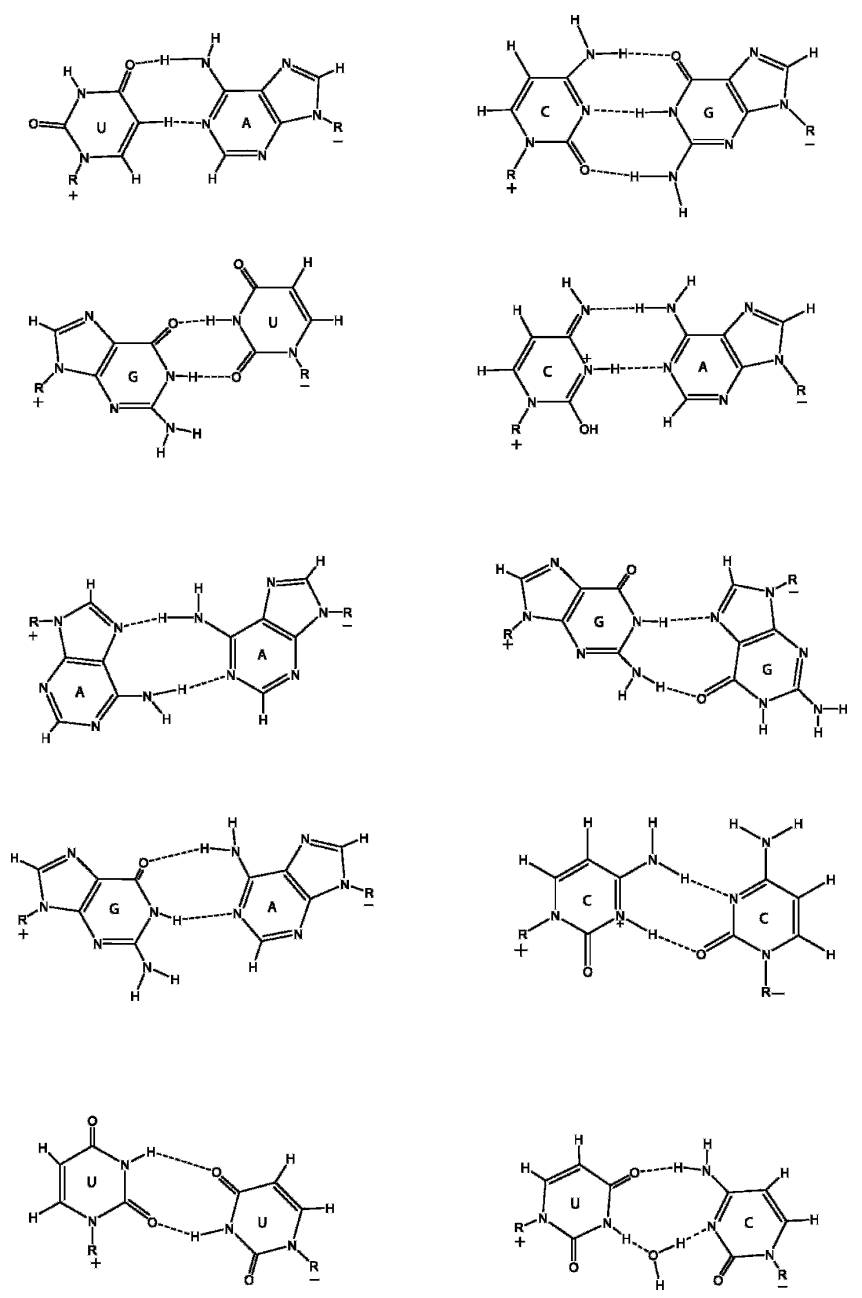

FIGURE 1. Proposed hydrogen bonding schemes for the eight noncomplementary antiparallel base pairs required if the stop codons used by different species interact with putative Ter-anticodons according to the model of Ivanov et al. (2001). Although there is more than one possible antiparallel arrangement for some base pairs, we show the arrangement that appears to be most compatible with the two complementary base pairs. All of these arrangements have been experimentally observed in rRNA or tRNA, except for the hemiprotonated C. $\mathrm{C}^{+}$pair. The Watson-Crick base pairs are shown on top for reference.

pairwise combination in at least one species for the TertRNA model to hold. Consequently, we considered how the eight noncanonical antiparallel base pairs, including one wobble $(G \cdot U / U \cdot G)$, one other purine.pyrimidine (A.C/ C.A), three purine purine (A.A, G.G, A.G/G.A), and three pyrimidine pyrimidine $(\mathrm{U} \cdot \mathrm{U}, \mathrm{U} \cdot \mathrm{C} / \mathrm{C} \cdot \mathrm{U}, \mathrm{C} \cdot \mathrm{C}$ ) pairs could contribute to stop-codon-Ter-anticodon specificity.

By all accounts, the two complementary base pairs are dominant: they are the most stable, and readily conform to a common helical array. The other eight base pairs have all been experimentally observed in crystal structures, either within or adjacent to antiparallel complementary duplexes (summarized in Nagaswamy et al. 2002). Each noncomplementary pair can form at least two hydrogen bonds in pairing arrangements, 
most of which have nearly standard glycosyl bond separation distances (Fig. 1; Donohue 1956). The question then is whether a variety of these base pairs can form three-base pair minihelices, stabilized within the ribosome A-site.

\section{Wobble G.U/U.G combination}

This antiparallel base pair is well known from standard codon-anticodon interactions, so it is the most easily accepted. It occurs in helices in solution (Strazewski et al. 1999) and in RNA duplexes bounded by complementary base pairs (Holbrook et al. 1991; Cruse et al. 1994) and has a glycosyl bond separation similar to that of a complementary base pair.

\section{A.C/C.A combination}

This antiparallel base pair requires protonation of either the A or the C residue (Topal and Fresco 1976a; Hunter et al. 1986a; Gao and Patel 1987; Purrello et al. 1993). It is possible that RF2 could promote A.C base pair formation with concurrent protonation of the $\mathrm{C}$ residue to form a wobble-type pair between stop codon and Ter-anticodon. The significantly higher $\mathrm{pK}_{\mathrm{a}}$ value of $\mathrm{C}$ (4.3) than of A (3.4) makes it more likely that the $\mathrm{C}$ residue is protonated (Fig. 1). In fact, a base pair with $\mathrm{C}^{+}$is isosteric with the standard wobble $\mathrm{U} \cdot \mathrm{G}$ base pair (Topal and Fresco 1976a). In addition, the macromolecular crowding inside the cell (Zimmerman 1993; J.R. Fresco, M. Ulasli, S. Kukreti, and O. Amosova, in prep.) makes such protonation seem especially likely.

Many Firmicute species have CCA at the Ter-anticodon sites, which require two sequential A.C pairs to recognize the UAA stop codon, however, which raises the energetic cost in a three-base pair miniduplex. Nevertheless, binding by a protein can drive the equilibrium distribution toward protonation of $\mathrm{C}$ residues very significantly.

\section{Purine.purine combinations}

All three antiparallel combinations, A.A, G.G, and A.G/G.A, have two hydrogen bonds and no protonated bases. Whereas a pair of purines in the anti configuration about the glycosyl bond requires a glycosyl bond separation $\sim 2.4 \AA$ longer than a complementary base pair, a pair with one base oriented syn and the other anti maintains the standard separation distance for a complementary pair, although there may be some distortion of the dihedral angles of the glycosyl bonds (Topal and Fresco 1976b; Patel et al. 1984; Hunter et al. 1986b; Dolinnaya et al. 1997; Nissen et al. 2001; Battle and Doudna 2002; Dolinnaya and Fresco 2003). The cost of forming such a base pair with a syn-oriented purine residue is hardly more than a kcal/mol (Topal and Fresco 1976b).

\section{Pyrimidine.pyrimidine combinations}

Antiparallel U.U and U.C base pairs have been observed in rRNA (Ban et al. 2000; Carter et al. 2000). Two crystallographic studies of RNA duplexes with U.C pairs bounded by complementary base pairs suggest that an interbase water bridge between the endocyclic $\mathrm{N} 3$ atoms of the $\mathrm{U}$ and $\mathrm{C}$ residues in the minor groove widens the glycosyl bond separation to approximately that of a complementary base pair (Holbrook et al. 1991). Such a water bridge could possibly have a comparable effect on the U.U and hemiprotonated $\mathrm{C} \cdot \mathrm{C}^{+}$pairs (Fig. 1) that also form at neutral $\mathrm{pH}$.

\section{Other relevant considerations}

While evaluating hairpin 69 and 89 sequences regarding stop codon usage, we made a number of additional observations that seem to question the validity of the Ter-tRNA model.

\section{Absence of hairpin 69}

The very short LSU rRNAs of kinetoplastid mitochondria lack hairpin 69. This leaves no apparent way to signal protein termination within the framework of the model.

Secondary structure variability in the regions of hairpins 69 and 89

Ivanov et al.'s model was inspired by the observation that hairpins 69 and 89 in E. coli, together with their neighboring sequences, mimic the cloverleaf secondary structure of tRNA. However, we found the size of the loops of these hairpins quite variable. For example, the loops of hairpin 89 in the mitochondrial LSU rRNA of kinetoplastids are usually only $4 \mathrm{nt}$, whereas that of Onchocerca volvulus are $8 \mathrm{nt}$. Moreover, in many species the secondary structures around these hairpins differ significantly from that of a tRNA cloverleaf. For hairpin 89, there are no neighboring hairpins mimicking the T $\Psi \mathrm{C}$ and DHU hairpins. Also, hairpin 70, which Ivanov and colleagues designated the TYC-stem-loop mimic (in E. coli) of the tRNA-like cloverleaf centered around hairpin 69 , is only $3 \mathrm{nt}$ in most other bacteria; in some species, e.g., the mitochondria of Loligo bleekeri, the size of hairpin 68 (the putative DHU stem-loop mimic) is much smaller than that in E. coli. Whether these variations are significant is unknown.

Apparent absence of conservation of Ter-anticodon sequences

If Ter-anticodons in hairpin 69 pair with the dominant UAA and UAG stop codons, one might expect them to be under strong selective constraint to maintain their most favorably pairing sequences. However, in very closely related Plasmodium species that contain fragmented mitochondrial LSU rRNAs (Gillespie et al. 1999), the Ter-anticodons lie on a conserved fragment but are very diverse in sequence (Table $3)$, implying lack of constraint in this region.

Ter-anticodon sequences in Firmicute LSU rRNAs

In some Firmicutes, the UGA stop codon is reassigned to tryptophan. We divided the 71 Firmicute species in the 
TABLE 3. Variation among closely related species of Plasmodium in Ter-tRNA1 anticodons of hairpin 69 in mitochondrial LSU rRNA

Hairpin 69

Species

P. berghei

P. chabaudi

P. falciparum

P. vivax

P. yoelii

${ }^{\mathrm{a}}$ Ter-anticodons are shown in uppercase letters.
rRNA database into two major groups, according to their UGA usage. We designate the 65 species that use UGA as stop, the Standard Codon Group, and the six remaining species that use UGA as tryptophan, the W Codon Group. Within the Standard Codon Group, 68\% have CCA at the putative Ter-anticodon site of Ter-tRNA2, 29\% have UCA, and $3 \%$ have a quadruplet Ter-anticodon, either UCCA or UCAA; within the W Codon Group, 100\% have UCA at the putative Ter-anticodon site.

Since the W Codon Group no longer uses UGA as stop, but nevertheless has $5^{\prime}$-UCA- $3^{\prime}$ at the Ter-anticodon site, the possibility for UGA stop codon.UCA Ter-anticodon interaction via three complementary base pairs still exists. Therefore, if the model is valid, there would seem to be a high risk of misreading UGA in these organisms. However, as Ivanov et al. (2001) remark, Trp-tRNA would always win in competition with Ter-tRNA, suppressing misreading of UGA as stop, because the process of codon recognition is much faster in elongation than in termination (Parker 1989). It is also possible that an unknown mechanism blocks binding between the stop codon and Ter-anticodon site in the W Codon Group.

Cross-linking does not seem to place the putative Ter-anticodons in close proximity to the termination site

A cross-linking study by Bulygin et al. (2003) tested whether the LSU rRNA of mammalian ribosomes could recognize a stop codon. Because the stop codon at the ribosomal A site cross-links to the $40 \mathrm{~S}$ (small) subunit and not to the LSU after mild UV irradiation, those authors concluded that the model does not seem relevant in such species. However, there was no direct comparison of Teranticodons and stop codons.

\section{DISCUSSION}

Although the structural and thermodynamic aspects of noncomplementary base-pairing may be compatible with the qualitative notion of stop codon-Ter-anticodon interaction, there are strong reasons to be skeptical of the TertRNA model. For one thing, studies in E. coli (Ito et al.
2000; Klaholz et al. 2003) indicate that tripeptide sequences of release factors RF1 and RF2 directly interact in some unknown way with stop codons in the ribosomal A site. The direct role of these release factors in stop codon recognition is not well understood. But the question raised by the Ivanov model is whether those release factors recognize the stop codons by themselves or in the context of stop codon. Ter-anticodon three-base pair minihelices. In contrast to the claim by Ivanov and colleagues that the model only requires A.C in addition to complementary and G.U wobble pairs, our study reveals a requirement for all eight noncomplementary base pairs, implying that all combinations of these pairs be able to form stable but stereochemically varied minihelices within the confines of the ribosome.

A serious problem arises if such interaction occurs in the ribosomal A-site, which imposes striking structural stringency on the sense codon.tRNA anticodon interaction to maintain fidelity of protein synthesis (Parker 1989), and only allows complementary base pairs in the first and second codon positions (Yusupov et al. 2001). If stop codon·Ter-anticodon interaction occurs within the same site, it is difficult to understand why the same machinery should tolerate so many noncomplementary bases pairs, even at first and second nucleotide positions of the stop codon. Also, with TertRNA in place of aminoacyl tRNA, it seems reasonable to expect that each of the Ter-anticodons ought to interact preferentially by complementary and wobble base-pairing with a particular sense codon in elongation, which could lead to a high risk of premature termination, although the downstream context of true stop codon would be lacking. And even if, as Ivanov and coworkers suggested, RF-1 and RF-2 do not interact directly with the stop codon alone, but instead recognize the minihelix between Ter-anticodon and stop codon, our findings suggest that the structural variability due to the range of noncomplementary base pairs in the minihelix would be too great to be a source of specificity. Since one would expect release factors to share a common cavity for sensing and stabilizing all stop codon-Ter-anticodon minihelices, the fact that the structures of these minihelices is so varied makes that possibility seem very unlikely.

Taken together, these considerations cast considerable doubt on the Ter-tRNA model, which we therefore consider highly speculative. Substantial experimental evidence is needed to lend the model credibility.

\section{MATERIALS AND METHODS}

Mitochondrial and bacterial 23S rRNA sequence alignments were downloaded from the comparative RNA Web site (www.rna.icmb. utexas.edu). After removing redundant sequences, there were 240 mitochondrial and 71 Firmicute sequences in the study. Sequences in hairpins 69 and 89 (Ivanov et al. 2001) of each species were then extracted from the sequence alignment by a PERL script. For those rRNAs with characterized secondary structures, the loop sequences were confirmed. 
GenBank annotation of stop codon usage was further confirmed with information from the codon usage database (http://www. kazusa.or.jp/codon/) (Nakamura et al. 2000). To be conservative, data for species whose stop codon usage is ambiguous [either because of a lack of cDNA data support, or because a stop codon is absent from the available data (if not all full genomes)] were excluded from the analysis.

\section{ACKNOWLEDGMENTS}

This work was supported in part by NSF grant DBI-9875184 to L.F.L.

Received April 12, 2005; accepted July 5, 2005.

\section{REFERENCES}

Ban, N., Nissen, P., Hansen, J., Moore, P.B., and Steitz, T.A. 2000. The complete atomic structure of the large ribosomal subunit at $2.4 \AA$ resolution. Science 289: 905-920.

Battle, D.J. and Doudna, J.A. 2002. Specificity of RNA-RNA helix recognition. Proc. Natl. Acad. Sci. 99: 11676-11681.

Bulygin, K.N., A Demeshkina, N., Frolova, L.Y., Graifer, D.M., Ven'yaminova, A.G., Kisselev, L.L., and Karpova, G.G. 2003. The ribosomal A site-bound sense and stop codons are similarly positioned towards the A1823-A1824 dinucleotide of the $18 \mathrm{~S}$ ribosomal RNA. FEBS Lett. 548: 97-102.

Cannone, J.J., Subramanian, S., Schnare, M.N., Collett, J.R., D’Souza, L.M., Du, Y., Feng, B., Lin, N., Madabusi, L.V., Muller, K.M., et al. 2002. The comparative RNA web (CRW) site: An online database of comparative sequence and structure information for ribosomal, intron, and other RNAs. BMC Bioinformatics 3: 2 .

Carter, A.P., Clemons, W.M., Brodersen, D.E., Morgan-Warren, R.J., Wimberly, B.T., and Ramakrishnan, V. 2000. Functional insights from the structure of the $30 \mathrm{~S}$ ribosomal subunit and its interactions with antibiotics. Nature 407: 340-348

Cruse, W.B., Saludjian, P., Biala, E., Strazewski, P., Prange, T., and Kennard, O. 1994. Structure of a mispaired RNA double helix at 1.6- $\AA$ resolution and implications for the prediction of RNA secondary structure. Proc. Natl. Acad. Sci. 91: 4160-4164.

Dolinnaya, N.G. and Fresco, J.R. 2003. Conformational polymorphism of $\mathrm{d}(\mathrm{A}-\mathrm{G}) \mathrm{n}$ and related oligonucleotide sequences. Prog. Nucleic Acid Res. Mol. Biol. 75: 321-347.

Dolinnaya, N.G., Ulku, A., and Fresco, J.R. 1997. Parallel-stranded linear homoduplexes of $d\left(A^{+}-G\right) n>10$ and $d(A-G) n>10$ manifesting the contrasting ionic strength sensitivities of $\operatorname{poly}\left(\mathrm{A}^{+} . \mathrm{A}^{+}\right)$ and DNA. Nucleic Acids Res. 25: 1100-1107.

Donohue, J. 1956. Hydrogen-bonded helical configurations of polynucleoties. Proc. Natl. Acad. Sci. 42: 62-65.

Gao, X.L. and Patel, D.J. 1987. NMR studies of A.C mismatches in DNA dodecanucleotides at acidic $\mathrm{pH}$. Wobble $\mathrm{A}$ (anti).C(anti) pair formation. J. Biol. Chem. 262: 16973-16984.
Gillespie, D.E., Salazar, N.A., Rehkopf, D.H., and Feagin, J.E. 1999. The fragmented mitochondrial ribosomal RNAs of Plasmodium falciparum have short A tails. Nucleic Acids Res. 27: 2416-2422.

Holbrook, S.R., Cheong, C., Tinoco Jr., I., and Kim, S.H. 1991. Crystal structure of an RNA double helix incorporating a track of nonWatson-Crick base pairs. Nature 353: 579-581.

Hunter, W.N., Brown, T., Anand, N.N., and Kennard, O. 1986a. Structure of an adenine-cytosine base pair in DNA and its implications for mismatch repair. Nature 320: 552-555.

Hunter, W.N., Brown, T., and Kennard, O. 1986b. Structural features and hydration of d(C-G-C-G-A-A-T-T-A-G-C-G); a double helix containing two G.A mispairs. J. Biomol. Struct. Dyn. 4: 173-191.

Ito, K., Uno, M., and Nakamura, Y. 2000. A tripeptide "anticodon" deciphers stop codons in messenger RNA. Nature 403: 680-684.

Ivanov, V., Beniaminov, A., Mikheyev, A., and Minyat, E. 2001. A mechanism for stop codon recognition by the ribosome: A bioinformatic approach. RNA 7: 1683-1692.

Klaholz, B.P., Pape, T., Zavialov, A.V., Myasnikov, A.G., Orlova, E.V., Vestergaard, B., Ehrenberg, M., and van Heel, M. 2003. Structure of the Escherichia coli ribosomal termination complex with release factor 2. Nature 421: 90-94.

Nagaswamy, U., Larios-Sanz, M., Hury, J., Collins, S., Zhang, Z., Zhao, Q., and Fox, G.E. 2002. NCIR: A database of non-canonical interactions in known RNA structures. Nucleic Acids Res. 30: 395-397.

Nakamura, Y. and Ito, K. 1998. How protein reads the stop codon and terminates translation. Genes Cells 3: 265-278.

Nakamura, Y., Gojobori, T., and Ikemura, T. 2000. Codon usage tabulated from international DNA sequence databases: Status for the year 2000. Nucleic Acids Res. 28: 292.

Nissen, P., Ippolito, J.A., Ban, N., Moore, P.B., and Steitz, T.A. 2001. RNA tertiary interactions in the large ribosomal subunit: The A-minor motif. Proc. Natl. Acad. Sci. 98: 4899-4903.

Parker, J. 1989. Errors and alternatives in reading the universal genetic code. Microbiol. Rev. 53: 273-298.

Patel, D.J., Kozlowski, S.A., Ikuta, S., and Itakura, K. 1984. Deoxyguanosine-deoxyadenosine pairing in the $\mathrm{d}(\mathrm{C}-\mathrm{G}-\mathrm{A}-\mathrm{G}-\mathrm{A}-\mathrm{A}-\mathrm{T}-\mathrm{T}-\mathrm{C}$ G-C-G) duplex: Conformation and dynamics at and adjacent to the dG X dA mismatch site. Biochemistry 23: 3207-3217.

Purrello, R., Molina, M., Wang, Y., Smulevich, G., Fossella, J., Fresco, J.R., and Spiro, T.G. 1993. Keto iminol tautomerism of protonated cytidine monophosphate characterized by ultraviolet resonance raman spectroscopy: Implications of $\mathrm{C}^{+}$iminol tautomer for base mispairing. J. Am. Chem. Soc. 115: 760-767.

Strazewski, P., Biala, E., Gabriel, K., and McClain, W.H. 1999. The relationship of thermodynamic stability at a $\mathrm{G} \times \mathrm{U}$ recognition site to tRNA aminoacylation specificity. RNA 5: 1490-1494.

Topal, M.D. and Fresco, J.R. 1976a. Base pairing and fidelity in codonanticodon interaction. Nature 263: 289-293.

. 1976b. Complementary base pairing and the origin of substitution mutations. Nature 263: 285-289.

Yusupov, M.M., Yusupova, G.Z., Baucom, A., Lieberman, K., Earnest, T.N., Cate, J.H., and Noller, H.F. 2001. Crystal structure of the ribosome at $5.5 \AA$ A resolution. Science 292: 883-896.

Zimmerman, S.B. 1993. Macromolecular crowding effects on macromolecular interactions: Some implications for genome structure and function. Biochim. Biophys. Acta 1216: 175-185. 

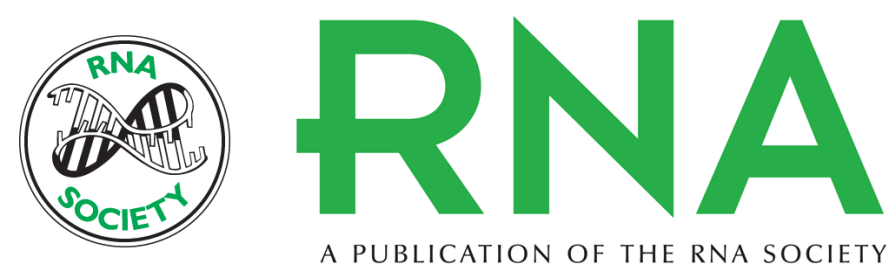

A PUBLICATION OF THE RNA SOCIETY

\section{Are stop codons recognized by base triplets in the large ribosomal RNA subunit?}

HAN LIANG, LAURA F. LANDWEBER and JACQUES R. FRESCO

RNA 2005 11: 1478-1484

References This article cites 29 articles, 9 of which can be accessed free at:

http://rnajournal.cshlp.org/content/11/10/1478.full.html\#ref-list-1

\section{License}

Email Alerting Receive free email alerts when new articles cite this article - sign up in the box at the Service top right corner of the article or click here. 